\title{
La educación en Colombia: mujeres en la escuela normal de institutoras de Bolívar (1903-1930)
}

\section{Education in Colombia: women in the normal school of Bolivar}

(1903-1930)

Yésica Paola Montes*

Universidade Federal de Mato Grosso

Nilce Vieira Campos Ferreira** Universidade Federal de Mato Grosso

Resumo Las escuelas normales femeninas en Colombia fueron creadas en el año de 1874 bajo el decreto $n^{\circ} 356$. En el departamento de Bolívar se creó la primera Escuela Normal de Institutoras de Bolívar en el año de 1878. Este artículo hace parte de una investigación que estudia la formación de profesoras en la escuela Normal de Institutoras de Bolívar en el periodo de 1903 a 1930 . Nuestro análisis tiene como fundamentos la historia de la educación a partir de la visión de la Historia Nueva, que introduce nuevos temas, objetivos, caminos, objetos y sujetos en las investigaciones históricas. Analizamos cómo se formaban las profesoras primarias a los inicios de siglo XX. La formación de maestras en las tres primeras décadas del siglo XX era considerada como el espacio de trabajo de la mujer colombiana.

PALAVRAS-CHAVE: Educación femenina; Escuela normal; Bolívar.

Abstract The normal feminine schools in Colombia were created in the year of 1874 under the decree $\mathrm{n}^{\circ} 356$. In the department of Bolivar, in the year of 1878, the first Normal School of Institutes of Bolivar was created. This article is part of an investigation that studies the training of teachers in the Normal School of Institutes of Bolivar in the period from 1903 to 1930 . This analysis is based on the history of education, based on the vision of New History, which introduces new themes, objectives, paths, objects and subjects in historical research. We analyzed how the primary teachers were formed at the beginning of the 20th century. The training of teachers in the first three decades of the twentieth century was considered as the workspace for Colombian women.

KEYWORDS: Women's education; Normal school; Bolívar. 


\section{Introducción}

Bien se ha dicho que la historia de la educación en Colombia ha estado marcada por los cambios políticos, económicos, sociales y culturales por los que ha atravesado el país, por lo que la formación de profesores y profesoras también ha variado de acuerdo a la época, los gobiernos, las leyes y el contexto local. Las Escuelas Normales, consideradas las escuelas de maestros, eran las encargadas de formar profesores y profesoras responsables de la educación pública nacional.

Groso modo, en Colombia, las Escuelas Normales nacen en el año de 1821, en las cuales se enseñaba el método lancasteriano ${ }^{1}$ que debía ser aplicado en las escuelas elementales. Sin embargo, en la segunda mitad del siglo XIX, bajo la reforma liberal, el analfabetismo y la irregular instrucción pública llegaban a ser uno de los problemas a reducir y para ello, se reformula la instrucción pública a través el primer Decreto Orgánico de Instrucción Pública en 1870 y se restablecen las Escuelas Normales como formadoras de maestros.

Uno de los puntos esenciales de la reforma de 1870, en materia de educación femenina, es la creación de las Escuelas Normales para mujeres, en las cuales se formarían las profesoras primarias y a la vez la mujer podría ocupar el oficio del magisterio. A partir de la Ley de 1870, la mujer comienza a ocupar el espacio público de la escuela como maestra primaria. Sin embargo, este proceso de feminización del magisterio no fue homogéneo, por el contrario estuvo lleno de combates, conflictos, dificultades y luchas de las mujeres por hacer parte activa de la sociedad.

En la ciudad de Cartagena se creó la Escuela Normal de Institutoras ${ }^{2}$ de Bolívar, en adelante ENIB, en el año de 1878, la cual había sido reglamentada en el Decreto ${ }^{\circ} 38$ de 1875. Esta institución funcionó de forma regular desde 1878 pasando por los altibajos que la Instrucción Pública colombiana sufría debido a las guerras civiles y los estragos en la economía nacional, los cuales harían que en algunas ocasiones la ENIB fuera cerrada.

Ya desde 1886 con la caída del liberalismo radical, los conservadores comenzaban a implementar una nueva idea de educación para el país, lo que implicaba una reforma a las leyes liberales. En 1903, la Ley de 1870 es revocada por la Ley n 39 o Ley Orgánica de Instrucción Pública, en la cual se intentaba establecer un sistema nacional de Instrucción Pública bajo las doctrinas católicas y la preparación del ciudadano para la industria, la agricultura y el comercio (OSPINA, 2010). De ahí, el siglo XX llega a Colombia marcado por las ansías de modernidad y progreso; la educación se regía bajo la Ley Orgánica de Instrucción Pública organizada por los conservadores y promovida por Antonio José Uribe, bajo la cual también se estableció el Ministerio de Instrucción Pública.

La nueva Ley Orgánica de Instrucción Pública de 1903 abolía la obligatoriedad de la educación primaria y la neutralidad religiosa, dos aspectos esenciales que fueron promovidos por los liberales en el Decreto Orgánico de Instrucción Pública de 1870. Estas ideas fueron reemplazadas por la imposición de la religión católica como base educativa y la continuación de la gratuidad de la educación primaria. 
Desde esta perspectiva proponemos un análisis desde Nueva Historia, la Historia de la Educación femenina y la Historia de las Instituciones Escolares para comprender las formas de instrucción que fueron pilares de la formación de maestras en el primer tercio del siglo XX en Cartagena-Bolívar. Por lo tanto, este artículo toma como objeto de estudio la Escuela Normal de Institutoras de Bolívar para entender la forma como se llevaba a cabo la formación de profesoras en el departamento de Bolívar-Colombia.

Así mismo, se enfoca en las primeras tres décadas del siglo XX, a partir de 1903, año en el que es reglamentada la Ley Orgánica de Instrucción Pública, firmada en el marco de una República conservadora que confía la Instrucción Pública a la iglesia católica y, concluye en 1930, fecha en la que el gobierno conservador pierde el poder del Estado y nuevas reformas educativas surgen en el país.

Las fuentes utilizadas comprenden dos tipos. Considerando los principios de Ester Buffa y Paolo Nosella (2009) sobre las posibles fuentes para estudiar instituciones educativas, se destacan las primarias y secundarias. El primer grupo abarca documentos históricos físicos y digitales como leyes, decretos, informes de instrucción, memorias y avisos oficiales sobre la educación de la época y la institución estudiadas encontrados en el Archivo Histórico de Cartagena, la Biblioteca del Banco de la República, sede Cartagena, el Centro de Memoria en Educación y Pedagogía de la Universidad Pedagógica Nacional y el Archivo General de la Nación -AGN. Como fuentes secundarias son utilizadas las tesis, artículos, libros e investigaciones enfocadas a temas como la Historia de la Educación, la educación femenina en Colombia, las escuelas normales, el contexto político, social, económico y cultural de finales del siglo XIX y la primera mitad del siglo XX.

Se inscribe desde la perspectiva teórica de la Historia de la Educación femenina, partiendo de los postulados de la Historia de la Educación con autores como Jane de Almeida (2004; 1998), Guacira Louro (2003), Miryam Báez Osorio (2000; 2002; 2004; 2005) y de la historia de la mujer con Michell Perrot (2009; 2006), Gloria Bonilla Vélez (2011), Rafaela Vos Obeso (1999). Ester Buffa e Paulo Nosella (2009), Jose D`Assunção Barros (2008) ,Justino Magalhaes (2004) e Giovanni Levi (1992), Jaques Le Goff (1990), nos trajeron los fundamentos metodológico a partir de la visión de la Historia Nueva, según la cual la historia debe ser escrita a partir de problemas y debe generar nuevas cuestiones.

\section{Fundamentos teóricos y metodológicos}

Hablar de mujeres, instituciones educativas y formación de profesoras implica traer, con una mirada del presente, la historia del pasado. En los años setenta, cuando el interés por estudiar la historia de las mujeres es imperante y los movimientos feministas toman poder, surge la posibilidad de analizar el pasado teniendo como protagonista al sexo femenino. Esta posibilidad comienza a ocupar espacios importantes en los campos de investigación, entre ellos en la Historia de la Educación, en la cual estudiar a la mujer era un camino necesario para conocer y entender las diversas formas, lugares y espacios de educación en una sociedad determinada. Por lo tanto, es posible afirmar que los estudios sobre Historia de la Educación femenina o de la mujer han crecido recientemente. 
Desde la óptica de Michell Perrot ${ }^{3}$, la historia de las mujeres está invisibilizada, oculta en una historia vista desde la masculinidad. Una historia que en sus inicios ve a la mujer como víctima para luego estudiarla como sujeto activo en la sociedad, otorgándole un papel en los espacios, no sólo privados, de los cuales ya se ocupaba, sino también, en los públicos (PERROT, 2009). Espacios públicos como las instituciones educativas, la labores en áreas de comercio y salud son algunos de los lugares en que la presencia del sexo femenino se posiciona a lo largo de la historia y le otorga un espacio de poder dentro de la sociedad.

En el avance de sus luchas para salir del espacio privado de actuación, las mujeres buscaron los espacios escolares. Al adentrarse a los espacios educativos, percibieron que uno de los primeros espacios públicos habilitados fuera del hogar sería el magisterio. En él, debido a la creencia del don inherente de maternidad, la mujer ocupaba el lugar de educadora para la infancia, siendo así, las instituciones educativas lugares de recibimiento para el sexo femenino y su educación. Sin embargo, la aceptación para educar a las mujeres no fue concebida en un estado de igualdad social, sino una lucha constante por los derechos de las mismas. Por lo tanto, al estudiar la Historia de la Educación femenina debemos analizar que así como existieron estados de opresión y sumisión que nos permiten entender la invisibilidad de la mujer, también es posible ver el sexo femenino desde la óptica de las vencedoras (ALMEIDA, 1998).

Consideramos que el magisterio sería a lo largo del siglo XX visto como un espacio de actuación propiamente femenino. Este fenómeno será denominado por autoras como Almeida (1998) y Veiga (2007) "feminización del magisterio", donde se le delega a las mujeres, en función de su género, las actividades del cuidado del hogar, la educación de las jóvenes generaciones. Comprendida de esta forma, cabría aun una remuneración menor para el oficio de enseñar cuando era ejercido por las mujeres.

Pensar la educación femenina nos remite a la historia de las instituciones educativas. Espacios construidos, en los cuales el ser humano es instruido de acuerdo con los sistemas educativos previamente elaborados y por lo menos pensados/traídos. Las instituciones educativas, tales como las conocemos, son orientadas para conducir la educación del ser humano en las fases de su desarrollo a lo largo de su vida (MAGALHÂES, 2004).

La escuela delimita espacios. Sirviéndose de símbolos y códigos, ella afirma lo que cada uno puede (o no puede) hacer, ella separa e instituye. Informa de los pequeños y grandes, de los niños y de las niñas. A través de sus cuadros, crucifijos, santas o esculturas, apunta aquellos/as que deberán ser modelos e permite, también que los sujetos se reconozcan (o no) en esos modelos. El edificio escolar informa a todos/as su razón de existir. Sus marcas, sus símbolos, sus arreglos arquitectónicos "tienen" sentido, instituyen múltiples sentidos, constituyen múltiples sujetos (LOURO, 2003, p. 58).

Las instituciones educativas, bien lo expone Louro (2003) son espacios, en los cuales se producen y reproducen prácticas escolares que crean diferencias entre seres humanos, sea por su sexo, raza, clase social o religión. Estas diferencias son evidentes en los planes de estudios, las normas de comportamientos, incluso los juegos permitidos a cada uno de los sexos y están motivadas por los cambios sociales, políticos, 
económicos y culturales. Así, analizar la historia de la ENIB nos permite comprender el perfil de la mujer que se formaba como profesora primaria en Cartagena, sus responsabilidades, los ideales que ne la época imperaban y los obstáculos vencidos.

Para ello, se inició un trabajo de campo dedicado a la recolección de documentos históricos, en el cual predominaron los textos escritos. La recolección de datos inicia como una experiencia de ensayo y error, un acto de palpar, ver, sentir y leer los monumentos, que para Le Goff (1990, p. 536) “[...] es todo aquello que puede evocar el pasado, perpetuar los recuerdos [...]" En esa línea de ideas, los monumentos son todos los lugares en los cuales los documentos del pasado están guardados. Los archivos de la ciudad como el Archivo Histórico de Cartagena -AHC- , la Biblioteca del Banco de la República -BBR-, el Centro de Memoria en Educación y Pedagogía de la Universidad Pedagógica Nacional y el Archivo General de la Nación -AGN- fueron los espacios en los que la búsqueda de documentos se hizo posible.

La metodología utilizada para el análisis de las fuentes encontrada en el archivos públicos de la ciudad de Cartagena se fundamenta en entender que la historia no puede ser vista como unitaria, estable y fija. Esta es el resultado de una mirada móvil de una realidad compleja, por lo que se debe atender a ver los cambios, asumir la realidad como un proceso (FRAGO, 1995). Así, la escritura de la historia, conocida como historiografía, la entendemos desde una posición dinámica, de miradas diversas, en las cuales la posición del escritor está sujeta a las fuentes y al objeto de estudio, para intentar llegar a entender la complejidad de la realidad desde perspectivas diversas (FRAGO, 1995). En palabras de Jose D’Assunção Barros (2008, p. 17) "la historia es siempre es múltiple, no obstante se la trate desde perspectivas diferentes.

Entendiendo la multiplicidad de los datos históricos, también se asume la multiplicidad de modos de estudiarla. Para la investigación tomamos los conceptos de dimensiones, enfoques y campos de investigación de Barros (2008) que permiten puntualizar hacia una óptica de estudio.

Una dimensión implica un tipo de enfoque o "un modo de ver" (o algo que se pretende ver en el primer plano de la observación de una sociedad históricamente localizada); un abordaje implica "un modo de hacer"la historia a partir de los materiales con los que debe trabaja el historiador (determinadas fuentes, determinados métodos y determinados campos de observación); un dominio corresponde a una elección más específica, orientada con relación a determinados sujetos y objetos a los cuales será dirigida la atención del historiador (campos temáticos como"la historia de la mujeres" o de "la historia del derecho) (BARROS, 2008, p. 20).

La historia de las instituciones educativas como dimensión; la Historia de las mujeres como dominio y la micro-historia como abordaje son los criterios utilizados para realizar la investigación, los cuales se construyen a partir de lecturas fundamentadas. La dimensión, considerada el criterio macro, es planteada desde los postulados de Ester Buffa, Paolo Nosella (2009) y Justino Magalhães (2004).

Nosella e Buffa (2009) nos indican que las investigaciones sobre instituciones educativas poseen un valor social y educativo para la comunidad en la que están 
inseridas. Así mismo, estos autores, nos ofrecen caminos para entender la importancia de las categorías de análisis, con las cuales estaremos trabajando en el tercer capítulo. Justino Magalhães (2004, p. 114) expone que "cada institución escolar o educativa integra ese todo más amplio que es el sistema educativo"; por tanto, debemos entender que la historiografía de las instituciones escolares no es un proceso aislado de los procesos culturales de la localidad.

La historiografía de la historia de las mujeres y su educación nos permitió estudiar el pasado. Jacques Le Goff (1990) apuntó sobre los fundamentos de la memoria colectiva como "instrumento y objeto de poder" (LE GOFF, 1990, p. 477), la cual es el espacio donde se manifiestan las identidades de la sociedad. Esa memoria permite al historiador conocer y estudiar la historia a partir de las modificaciones de la misma memoria. Según Ana Carolina Ibarra (2007, p. 4) "para Le Goff la memoria es la materia prima del historiador". Es a partir de la memoria colectiva hallada a través de los documentos históricos que pretendemos entender la historia de la educación femenina en Bolívar y en la ENIB.

Así mismo, fundamentamos nuestro estudio en algunos teóricos de la Nueva Historia como Peter Burke (1992), Giovanni Levi (1992), Jacques Le Goff (1990) quienes posibilitaron la comprensión de la historiografía como un estudio más allá de las historia de los héroes. Una historia basada en el contexto social y cultura que enmarca a todos los sujetos. La microhistoria como abordaje posibilitó un estudio desde la "disminución de la escala de observación, en un análisis microscópico y en un estudio intensivo documental” (LEVI, 1992, p. 137). Por lo tanto, a micro-historia nos permitió estudiar un objeto particular sin olvidarnos de su contexto global, utilizando para ello el análisis de documentos históricos.

La microhistoria se funda en el principio de que la elección de cierta escala de observación reducida puede ser instrumento válido de una particular estrategia de conocimiento científico. Por otro lado, la microhistoria no es, y no puede ser una mera verificación de reglas macrohistóricas generales, de modo que no puede propiciar ejemplo de los datos ya constatados a nivel global ( MAN, 2013, p. 169).

La microhistoria pretende salir del dualismo binario entre el la historia totalizadora y la historial particular, es decir, mirar el objeto de estudio desde su particularidad sin dejar de entender que está inserido en un espacio global que lo influye. No existe una historia total y fija, globalizadora, existen realidades y miradas microscópicas que posibilitan la construcción de nuevas teorías y nuevos acercamientos con el pasado.

La Micro Historia como enfoque metodológico es fundamentan por dos razones. La primera de ella, es la delimitación de un espacio tiempo para entender la educación femenina dentro un momento histórico específico, en un espacio específico. La segunda y más esencial, es permitir observar y comprender elementos dentro de la Institución educativa que posibilitan entender la sociedad más amplia. Esto es, elementos de la Escuela Normal de Institutoras de Bolívar para comprender la formación de profesoras en la ciudad. Por lo tanto, al trabajar desde la Micro Historia no sólo reducimos el espacio y el tiempo, sino la escala de observación para, a partir de elementos particulares, entender una realidad histórica y construir la memoria colectiva. 


\section{Creación de la escuela normal de institutoras de Bolívar}

Educar a la mujer implicaba crear instituciones educativas femeninas acordes con los saberes pedagógicos, sociales y culturales que para la época las mujeres debían aprender con el objetivo de aplicarlos en su vida futura, lo cual predeterminaba su papel social. Para ello, se aumentan las escuelas públicas para niñas y se crean las Escuelas Normales de mujeres, que irían a formar a las profesoras colombianas.

Para la fecha de 1875, existían en el Estado Soberano de Bolívar ${ }^{4}, 25$ escuelas privadas y públicas para la educación femenina, tal como lo expone el entonces director general de Instrucción Pública del Estado de Bolívar en su informe anual,

$2^{\circ}$ De niñas. Las siguientes: cartajena (dos), Villanueva, Mahates, Barranquilla (dos), Soledad, Santo Tomás, carmen, Corozal, Chinú, Lorica, Magangue, Mompos, Sabanalarga i Sincelejo. Total 16 escuelas. Después de la fecha indicada, se han abierto las escuelas de varones de Turbaco i Yati, i la de niñas de Sahagun, lo que da por resultado 58 escuelas de varones y 17 escuelas de niñas, que hacen un total de 75 escuelas con 3.395 alumnos matriculados. [...] Hai establecimientos de educación privada para niñas, en los lugares siguientes: en Cartajena, " 2 ", colejio del Carmen", a cargo de la señora J. B. Fernández de Araujo i el señor Juan Antonio Araujo; "Academia de instrucción privada" a cargo de las señoritas Eugenia i Zoila Moré; "Academia de niñas" a cargo de la señorita Margarita Bravo;en Barranquilla "colejio de María" a cargo de la señora Carmen santodomingo Vilas; - en el Carmen sin nombre especial, a cargo de la señora Francisca Gonzales del Cartillo; en el Guamo, id. a cargo de la señora Dolores Gutierrez de De Juano;- Sambrano, id. a cargo de la señora R. De trinidad Blanco; i Sampués, id. a cargo de la señora R. R. Martínez e señorita Rosa Verbel. Total de niñas matriculadas, 239 (BOLÍVAR, 1875, p. 804).

Por su parte, la formación de profesoras en Bolívar dependía de la creación de la ENIB. Según el Decreto n 38 de 1875, creado en virtud de lo establecido en el Decreto n 356 de 1874, se establecería la primera Escuela Normal para formar maestras en el Estado de Bolívar, la cual estaría ubicada en Cartagena, capital del Departamento. No obstante, en los estudios historiográficos referentes a educación femenina, en los que se analiza la fecha de apertura de la ENIB, se presentan algunas incongruencias con relación a la fecha de apertura.

Miryam Báez, es una de las autoras colombianas que investiga sobre las Escuelas normales de Colombia, expone que la ENIB “inició tareas en la ciudad de Cartagena en septiembre de 1878, con mucha alegría porque portaba los mejores anhelos de servicio educativo hacia el sector femenino" (OSORIO, 2002, p. 6). Sin embargo, otros autores manifiestan que la ENIB fue creada en 1875.

En el Estado de Bolívar se crea el 9 de marzo de 1875 por medio del Decreto 356 de 1874. La escuela fue iniciada bajo la dirección de la señorita Zoila Eugenia Moré, y desde el principio pudo contar con 6 alumnas externas oficiales, 3 internas y 3 asistentes, mientras que la escuela Anexa contaba con 57 estudiantes (OSORIO, 2005, p. 52). 
En la cita anterior podemos apreciar que algunos estudios afirman que la ENIB se crea en 1875. Ello, como consecuencia del Decreto $n^{\circ} 38$ que se establece y publica en el periódico oficial en el año de 1875. Si bien este reglamento, que está en conformidad con el Decreto ${ }^{\circ} 356$ de 1874, estipula, en su primer artículo, la creación de la Escuela Normal de Institutoras de Bolívar en la capital del Estado, no se tiene pruebas que la apertura de la Escuela Normal se realizara conforme a lo manifestado en él.

Ahora bien, en el Decreto n 38 de 1875 encontramos dos artículos referentes a las condiciones de apertura de la ENIB.

Artículo 161: el presente decreto tendrá vigencia el $1^{\circ}$ de mayo próximo, si fuere aprobado por el Poder Ejecutivo Federal. Artículo 162: sin embargo de lo dispuesto en el artículo anterior, se procederá a contratar el arriendo del local, la construcción o compra de útiles i mobiliario, para que puedan abrirse los cursos en el día indicado (BOLÍVAR, 1875, p. 258).

Según el artículo n 161 y n 162 la ENIB debía iniciar sus labores el $1^{\circ}$ de mayo de 1875 , lo cual estaría condicionado a la disponibilidad de las instalaciones. Esto quiere decir que la apertura de la ENIB dependería de la adecuada gestión y construcción del local, en el cual funcionaría la institución educativa; o en caso contrario, del arriendo de un local y la construcción del mobiliario para la escuela. Sin embargo, la prensa local no manifiesta haberse gestionado tales acciones.

Así mismo, en el informe anual sobre Instrucción Pública del Estado de Bolívar dado el 16 de agosto de 1875 por el entonces director general de Instrucción Pública M. Verbel, expone los avances de la Instrucción Pública del Estado de Bolívar, realizando un balance sobre las escuelas públicas y privadas, en cual resalta la labor de la Escuela Normal de institutores, el Colegio Pinillos y la academia del Bello Sexo; mas no ofrece información relacionada con la ENIB.

Posiblemente, la causa de la tardía instalación de la ENIB sería la falta de recursos locales debido a la crisis económica de Bolívar en la época y la guerra civil que inició en 1876 y acabó en 1877. Según Osorio ( 2005) las guerras civiles, en la segunda mitad del siglo XIX entre liberales radicales y conservadores, implicaban en algunas ocasiones el cierre de las instituciones escolares. Ello, sucedió en el Estado de Bolívar en el 1876, como se evidencia en un anuncio de la prensa local“la guerra, terrible calamidad que aflige a los pueblos, hizo cerrar las escuelas. Las del distrito de esta provincia fueron clausuradas en Diciembre último"(BOLÍVAR, 1877, p. 464).

Fue en 1878, tres años después de publicado el Decreto n ${ }^{\circ} 38$, que se abren las puertas de la ENIB en la ciudad Cartagena. Así se manifiesta en la prensa de la época en una nota que envía el gobernador de Cartagena el día 6 de enero de 1878,

Tengo el honor de decirle a ud que el día 6 del presente, a las doce del día, se verificó la inauguración de la Escuela Normal de Institutoras de esta ciudad, cuyo acto presidí en cumplimiento de la delegación que el C. Presidente se sirvió conferirme y que me comunicó a Ud. en nota $n^{\circ} 27$, fecha 6 del mismo mes, Sección del gobierno. De Ud. atento servidor, Ignacio G. Guerra (BOLÍVAR, 1878, p. 886). 
En el Estado de Bolívar fueron 3 las mujeres admitidas en la ENIB como alumnas-maestras externas y 6 más mostraron en los exámenes de admisión que necesitan mayores estudios para ingresar al plantel. Para el inicios de 1879, según el informe del director de Instrucción Pública primaria, en el departamento de Bolívar, existía una Escuela Normal con 8 alumnas pensionas, 3 externas y 3 asistentes. Sin duda alguna, el reducido número de estudiantes normalistas y las dificultades de mobiliario ${ }^{5}$ para la escuela hacían de esta tarea todo un reto. No obstante, para el 1880, ya se encontraban 12 escuelas normales de varones y 10 de mujeres en todo el país (OSORIO, 2005, p. 431), entre ellas la ENIB.

No cabe duda que la segunda mitad del siglo XIX marcó la historia de la educación femenina con la apertura de las Escuelas Normales femeninas en todo el país, constituyendo una nueva forma de formación y empleo para las mujeres de clase media, a través del cual conseguirían una nueva forma de ingresar al espacio público. Las mujeres normalistas estaban caracterizadas por dos razones: aquellas que sus padres podían costear sus gastos escolares e ingresaban a la institución como alumnas internas y aquellas en condiciones de pobreza que al obtener una beca nacional o departamental iniciaban su formación normalista.

No obstante, ambas mujeres debían presentar exámenes que demostraran conocimientos básicos de lectura, aritmética, escritura, religión, castellano y geografía. Las mujeres normalistas eran esas jóvenes que desde tiempos anteriores estaban destinadas al trabajo de casa, la obediencia al padre y al esposo, el cuidado de hijos y que desde 1874 podrían desempeñar el papel de educar en las escuelas.

\section{La escuela normal de institutoras de Bolívar (1903-1930)}

Con la posesión del conservadurismo en el gobierno colombiano, se inicia lo que se conoce como Hegemonía Conservadora. Una época en la que la Iglesia católica recupera el lugar que con el liberalismo radical se le había disminuido. A nivel educativo, la Instrucción Pública se rige en concordancia con la religión católica ${ }^{6}$, lo que le exige a las instituciones educativas reorganizar las disciplinas escolares e incorporar cátedras de religión obligatorias y orientadas por un sacerdote.

A partir de allí, se realizan cambios sustanciales en la educación colombiana partiendo desde la abolición del laicismo, el refuerzo de la relación Estado-Iglesia y la educación técnico-profesional.

La Ley orgánica a fue nos estamos refiriendo estableció la debida armonía entre los derechos del individuo, de la familia de las corporaciones, del Estado y de la Iglesia, así como el conveniente consorcio entre las actividades del Municipio, del Departamento y de la Nación, para trabajar de consumo; la obra múltiple y fecunda de la instrucción y de la educación del pueblo (COLOMBIA, 1927, p. 8).

Para Antonio José Uribe, entonces ministro de Instrucción Pública y promotor de la Ley 39 (Ley Orgánica de Instrucción Pública o ley Uribe), la Ley de 1903 era la reforma educativa más importante que se debía realizar en Colombia. Una reforma que estuvo vigente hasta 1994 cuando se crea la Ley General de Educación. 
La Ley n 39 insistía en una educación práctica, para el ejercicio de la ciudadanía y la preparación para la agricultura, la industria y el comercio (OSPINA, 2010). En ella, se establece que las Escuelas Normales deberían formar profesores y profesoras en las nociones fundamentales aplicables a la industria, a la agricultura y al comercio que deban ser transmitidos a los niños (artículo 15). La formación en las Escuelas Normales debía centrarse en la práctica y la pedagogía del conocimiento, más que a la transmisión del mismo.

En la educación femenina, guiada por el catolicismo de la época, se reafirmaba aún más la imagen de la mujer como ser de bondad y caridad que debía estar al cuidado del prójimo, asociado a la imagen Cristiana de la virgen María (OBESO, 1999). La mujer se educaba para que cumpliera con las labores del hogar, se casara y pudiera mantener la familia unida. Así, como expresa Rafaela Vos Obeso (1999, p. 107), "la pedagogía católica relevó la educación laica, promocionada por el viejo radicalismo, y las rígidas normas morales continuaron regulando la institución familiar [...]".

El aprendizaje de las buenas maneras era también parte del programas de los colegios femeninos así como ciertas actividades manuales nobles; las alumnas aprendían a cortar, a coser y a bordar, en particular vestidos de recién nacidos y niños [...] Fuera de esto, los establecimientos secundarios continuaban la instrucción básica dada en la escuela primaria: lengua materna, matemáticas, historia, geografía, disciplinas sobre las que se apoyaba la pedagogía, que constituía la orientación principal de la enseñanza femenina [...] (HELG, 1987, p. 86).

La instrucción femenina estaba dirigida para la formación de la mujer como protectora del hogar, edificadora de amor y responsable por las labores domésticas así como sinónimo de pureza, bondad y caridad. La mujer y su labor social estaban marcadas por los ideales conservadores hacia la construcción de una nación, en la cual el progreso iba de la mano con el catolicismo y el hombre trabajador junto a la mujer hogareña conformaban el ideal de familia.

La mujer debía ser formada para una vida de castidad, obediencia y sumisión. Una mujer preparada para el trabajo del hogar no debía tener acceso a la misma educación masculina, puesto que el hombre se educaba para la vida pública y ella para la vida privada. La educación femenina estaba dirigida para preparar a la futura esposa y madre, considerándose ese su rol social eminentemente natural.

La imagen heredada del pasado de la mujer como "bien sagrado" (virgen, madre y religiosa) o como objeto sexual, encubierta también por la maternidad y pureza, permanecía en la ideología social, imponiéndole un código moral que contribuía al conservadurismo cultural. El acervo religioso configuró los imperativos de la época, determinando los patrones, costumbres y valores que pesaban sobre las mujeres de ese momento (OBESO, 1999, p. 71).

En las Escuelas Normales la formación, tanto de maestros como de maestras, también se dirigía hacia el catecismo y moral. Fueron agregadas las disciplinas de religión, las cuales eran administradas por sacerdotes. Así, lo estipula la reforma hecha el 15 de junio de $1888^{7}$, en la cual la disciplina de religión es reglamentada en todos 
los cuatro años de estudio con una intensidad horaria de tres horas semanales (BOLÍVAR, 1888). Así mismo, es interesante anotar cómo en el artículo $3^{\circ}$ y $7^{\circ}$ de la misma reforma se establecen reglas de exclusividad para las Escuelas Normales femeninas.

Artículo $3^{\circ}$. En las Escuelas normales de Institutoras se darán las mismas enseñanzas de en las de varones, con excepción de Agricultura, contabilidad oficial y física; y en lugar de estas se darán las de modistería, costura y arte de la cocina. Artículo $7^{\circ}$ Las alumnas de las Escuelas normales no podrán salir del Establecimiento en los días feriados. La Directora podrá otorgar a una o más alumnas, en calidad de premio, permiso para salir hasta dos distintos al mes, siempre que no sean feriados, debiéndose tomar las precauciones necesarias, para que fuera del Establecimiento observen buena conducta. (BOLÍVAR, 1888, p. 266).

Analizando la legislación, podemos reflexionar sobre la educación pensada para la mujer colombiana a partir de algunos puntos descrito en los artículos $3^{\circ}$ y $7^{\circ}$. Confirmamos que una formación considerada como representación ideal de una mujer educada debería estar asociada a la idea de educar para el trabajo del hogar y la moral de la infancia. El primero de ellos, es el ideal de la formación femenina en la época, el cual consistía en educar mujeres esposas y madres. Para que la mujer se desempeñara de esta forma su educación debía estar orientada hacia las labores del hogar o aquellas que no le exigieran separarse de sus padres o en el futuro de sus hijos y esposo. Para Gloria Bonilla Vélez (2011) el trabajo fuera del hogar no era bien visto por los sectores dominantes a finales del siglo XIX y comienzos del XX. Aún persistía la resistencia hacia el cambio del rol femenino, y las instituciones educativas, en concordancia con las doctrinas católicas, educaban para hacer de la mujer un ser dedicado al hogar.

Así, no era de extrañar que en las Escuelas Normales existieran cátedras de cocina, costura y modistería que reemplazaran las cátedras de agricultura y física dictadas en las de varones, para formar maestras capaces de inculcar en la niñez femenina la futura labor de la mujer. No obstante, Bonilla (2011) manifiesta que fue la pobreza y los avances en el campo de la industria lo que permitió que la mujer, desde el siglo XX, su incluya en áreas de trabajo diferentes al magisterio y la enfermería.

Otro punto a resaltar del artículo tres, es la inclusión de estas disciplinas con la reforma conservadora. En el Decreto n 38 de 1875 en el capítulo 12, que establece la división y duración de los cursos en la ENIB, no se estipula cátedras de cocina, costura y modistería así como tampoco existe la disciplina de religión en la formación de profesoras. Sin embargo, saber coser era uno de los requisitos a cumplir. Esto puede interpretarse como la búsqueda por el ideal de maestro en la reforma radical. La construcción de un maestro laico, "modelo de las buenas maneras y de las virtudes ciudadanas" (LOAIZA, 2007, p. 83) que a diferencia del ideal conservador formaba bajo el catecismo, las nuevas industrias y el ideal de progreso.

Con respecto al artículo $7^{\circ}$ es preciso resaltar la importancia que se le otorga a mantener y vigilar la conducta de las alumnas-maestras. Siendo las mujeres reflejo de obediencia y castidad, no podían la Escuelas Normales permitir que sus alumnas mostraran en su vida privada o pública señales de una conducta considerada inadecuada. 
Por lo tanto, se restringía a las alumnas internas salidas sin autorización de la directora del plantel. Este reglamento también existió con la reforma liberal, en la que se exigía ser de buenas costumbres para poder ser estudiante de la Escuela Normal. Carlos Ospina (2011) manifiesta que esta exigencia no sólo afectaba a las estudiantes de las Escuelas Normales, sino a todas las profesoras de la época, a quienes se les prohibía casarse si asumían el oficio del magisterio.

Ahora bien, el Decreto $n^{\circ} 491$ de 1904, el cual reglamentaba las disposiciones sobre la Ley n ${ }^{\circ} 39$, contiene en el capítulo I del título XII las normas sobre las Escuelas Normales. En él, se reafirma que la formación de profesores y profesoras debe realizarse en cuatros años (artículo 115). Con relación a los nuevos planes de estudio de las Escuelas Normales, en el ya denominado departamento de Bolívar ${ }^{8}$, en el año 1905 se publicaba en la prensa local el Decreto n 194 que establecía las disciplinas de estudio de las Escuelas Normales de la cuidad, divididas en los cuatro años de estudio. Para la ENIB se estipulaba:

Quadro 1 - Plan de Estudio, 1905

\begin{tabular}{|c|c|c|c|c|}
\hline \multicolumn{5}{|c|}{ Años } \\
\hline & 1 & 2 & 3 & 4 \\
\hline \multicolumn{5}{|c|}{ Horas Semanales } \\
\hline Religión y Moral & 3 & 3 & 3 & 3 \\
\hline Pedagogía teórica & 3 & 3 & 3 & 3 \\
\hline Pedagogía práctica & & & 3 & 3 \\
\hline $\begin{array}{l}\text { Idioma nacional (lectura, } \\
\text { gramática, ortografía, ejercicios } \\
\text { de redacción y elocución) }\end{array}$ & 3 & 3 & 3 & \\
\hline $\begin{array}{c}\text { Aritmética teórica y } \\
\text { práctica y sistema legal } \\
\text { de pesos y medidas }\end{array}$ & 3 & 3 & 3 & 3 \\
\hline Contabilidad & & & & 3 \\
\hline Escritura & 3 & 3 & 3 & 3 \\
\hline Geografía de Colombia & 3 & 3 & & \\
\hline Geografía general y cosmografía & & 3 & 3 & \\
\hline Historia universal & & & 3 & 3 \\
\hline Historia de Colombia & & 3 & 3 & 3 \\
\hline Higiene & & & & 3 \\
\hline $\begin{array}{l}\text { Nociones elementales de ciencias } \\
\text { naturales, horticultura y economía } \\
\text { doméstica }\end{array}$ & 2 & 2 & 2 & 2 \\
\hline Dibujo, formas geométricas & 3 & 3 & 3 & 3 \\
\hline Costura y corte & 3 & 3 & 3 & 3 \\
\hline Música teórica y práctica & 3 & 3 & 3 & 3 \\
\hline Calisténica y gimnacia & 3 & 3 & 3 & 3 \\
\hline
\end{tabular}

Fonte: Registro de Bolivar, 1905

El plan de estudio para la ENIB presentaba algunos rasgos diferenciales con relación al contemplado para la Escuela Normal de Varones. Además de cursar cuatro cursos menos que la Escuela Normal para hombres, en la ENIB se ofrecían clases de costura, corte y economía doméstica en todos los años de formación de las profesoras. Contrario a esto, los alumnos-maestros recibían clases de arboricultura, ál- 
gebra, nociones elementales de preceptos constitucionales y administrativas, geometría plana, espacial y agrimensura, mientras que las alumnas-maestras recibían clases de dibujo y formas geométricas.

Estos planes de estudio cumplían con las exigencias que la Ley n 39 y el Decreto $n^{\circ} 491$ reglamentaban para formar los nuevos profesores del siglo XX. Sin embargo, también son muestra que en la época la formación de profesoras estaba unida al patrón femenino de trabajadora de casa, hacedora de actividades directamente relacionadas a espacio privado del hogar.

Las maestras y las enfermeras estaban asociadas con el "don de cuidar”, que se le atribuye a las mujeres. Las primeras atendía a los niños y las segundas al conjunto de la población [...] Maestras y enfermeras hicieron realidad la idea de que la mujer tenía la misión de cuidar y consolar a los que las rodeaban. Este hecho ocurrió en un contexto en el cual algunos políticos colombianos de finales del siglo XIX, basándose especialmente en la experiencia de Estados Unidos, consideraban que las soltera eran las indicadas para educar a los jóvenes (VÉLEZ, 2011, p. 182,183).

Como lo manifiesta Gloria Bonilla Vélez (2011) las mujeres y su rol en el magisterio estaban asociadas a la idea de madres educadoras, transmisoras de valores y veedoras de las buenas conductas del estudiantado, lo que hacía ver al magisterio femenino como una prolongación del hogar. De igual forma, Nilce Vieira Campos Ferreira (2014), al estudiar los programas del curso de economía rural doméstica en Uberaba-Brasil, identifica que los cursos relacionados a economía doméstica tenían el objetivo de educar a la mujer para el trabajo del hogar, colocándola como la responsable por la armonía y el bienestar familiar.

A partir de ello, podemos identificar dos puntos relevantes. El primero ligado a la idea de maternidad, que implicaba una formación fundamentada en los conocimientos básicos para el cuidado del hogar, por lo que las disciplinas de costura, corte y economía doméstica eran indispensables dentro del currículo de la ENIB. El segundo, está relacionado a la transmisión de ese conocimiento a las nuevas generaciones; la mujer como profesora del sexo femenino debía inculcar en sus estudiantes los saberes necesarios para que las niñas, y por ende futuras mujeres, conocieran y desempeñaran su rol social de forma adecuada.

La disciplina de arboricultura presente en el currículo de la Escuela Normal de Varones era sustituida por la de economía doméstica en la ENIB. Heloisa de Oliveira Santos Villela (2007) resalta que en Brasil, al igual que en Colombia, los currículos de las escuelas masculinas y femeninas, incluyendo los de las escuelas normales se diferenciaban, especialmente en las áreas de matemáticas y geometría, para las cuales las mujeres tenían un acceso limitado y casi nulo. Villela (2007, p. 109) afirma que principalmente la disciplina de matemáticas "era la ausencia más imperante, nunca yendo más allá de las operaciones simples".

Esta diferenciación es explicada por Obeso (1999) como una muestra que la educación femenina, además de no ser un tema prioritario en la época y no poseer 
los mismos planes de estudios que las escuelas masculinas, se dirigía expresamente a formar futuras madres y amas de casa, lo que hacía que el Estado le restaba importancia a la formación de la mujer.

Los cursos de matemática, geometría y álgebra en la ENIB eran reemplazados por disciplinas de corte y costura, los cuales aunque no habían sido exigidos en el Decreto ${ }^{\circ} 38$ de 1870, comienzan a ser obligatorias en el año de 1905. Para poner en marcha estas áreas de conocimiento, se crea para la ENIB, en el año de 1905, el Decreto $n^{\circ} 254$ de 29 de abril, en el que se estipula la implementación del curso de corte y literatura. Esta disciplina era ofrecida para las estudiantes más adelantadas; sin embargo, existía un curso de costura y corte que era obligatorio para todas las alumnas-maestras y correspondía a tres horas semanales por los cuatro año de formación, lo cual reafirma que la educación femenina basada en los patrones de cuidado, protección y abnegación al hogar se reflejaba en los cursos administrados, no solo en las escuelas primarias de niñas, sino en las escuelas normales.

Para el año 1906, la ENIB contaba con 24 alumnas en la escuela superior y 55 en la Anexa y las aulas se realizaban de acuerdo a lo estipulado en la Ley n 39 y los Decretos n 491 de 1904 y n 194 de 1905, con excepción de la clase de música, puesto que la profesora encargada había renunciado (BOLÍVAR, 1905). Sin embargo, los obstáculos eran recurrentes, como se puede apreciar en el informe mensual del director de Instrucción Pública del departamento sobre la visita hecha a la institución.

\begin{abstract}
Se tomó nota de la petición hecha por la señora Directora para que se cree un empleo de sirviente que pueda atender el aseo del local, á la provisión de agua en los depósitos y también para que se provea del gasto del alumbrado que hasta ahora está costeando la misma Directora, pues estos gastos estaban antes inclusos en el contrato de alimentación que ha cesado. [...] La señora Directora hizo saber que las 24 alumnas de la escuela secundaria actuales continúan como internado la mayor parte de ellas, con la diferencia de que los alimentos se los envían de la casa [...] (BOLÍVAR, 1906, p. 326).
\end{abstract}

Los alimentos no suministrados en la institución y la falta de personal de aseo para la ENIB eran algunos de los problemas que se presentaban a inicios del año 1906. Los desafíos de la entonces directora, María América Blanco de García, no solo consistían en vigilar la adecuada formación de las alumnas-maestras, sino en solucionar, si era posible con su dinero, las necesidades de la institución. No obstante, la ENIB continuaba sus labores formativas los años siguientes.

Otro aspecto importante dentro del análisis eran los salarios de los maestros. Para el año de 1909 se resolvió en el Decreto n 131 que cada Escuela Normal contaría con un personal conformado por un director, un subdirector, dos celadores, un portero y cuatro profesores.

Artículo $2^{\circ}$ : el personal de todas las Escuelas Normales, excepto la de Bogotá, constará de: Un Director Profesor, con ochenta pesos (\$80) oro de sueldo mensuales, además de una clase remunerada y una pensión alimenticia; Un Subdirector Profesor, con cuarenta y cinco pesos (\$45) oro mensuales, con derecho a una clase remu- 
nerada y pensión alimenticia; Cuatro Profesores más, con sueldo de veinte pesos $(\$ 20)$ oro mensuales, clase diaria; Dos celadores, a cuarenta pesos (\$40) oro mensuales y pensión alimentaria; Un Portero, a doce pesos y cincuenta $(\$ 12,50)$ oro mensuales y pensión alimentaria [...] (BOGOTÁ, 1909, p. 50).

Si se analiza detalladamente el artículo 2 del Decreto n 132 de 1909, los profesores y profesoras de las Escuelas Normales poseían un salario de 20 pesos oro, el cual era correspondiente a la cuarta parte del director, quien obtenía un sueldo de 80 pesos mensuales. Así mismo, resultaba ser el segundo sueldo más bajo después del de los porteros que ganaban 12 pesos con 50 centavos. Los profesores de las Escuelas Normales, además de tener el segundo bajo salario de los empleados, no obtenían pensiones alimentarias.

Ello, es muestra de que a pesar de los esfuerzos por mejorar las condiciones de la Instrucción Pública colombiana, en las Escuelas Normales, y probablemente en mayor medida en las escuelas primarias, existían un desbalance en los salarios, lo que posiblemente limitaba la voluntad de los profesores para ejercer el magisterio. Así mismo, la escasez de mobiliario, la falta de edificaciones para las escuelas, los pocos útiles escolares, incluso el alto número de maestros no graduados impedía que la Instrucción Pública en todas sus modalidades cumpliera con las metas propuestas. Estos obstáculos eran recurrentes en todo el periodo de estudio.

$\mathrm{Al}$ respecto de la carencia de edificios propios y aptos para las Escuelas Normales, se expone:

Una de las necesidades más urgentes e imperiosas que tienen las Escuelas Normales es la construir edificios amplios, cómodos e higiénicos, donde puedan funcionar con holgura. Ya se ha dicho que la mayor parte de los locales que ocupan aquellos planteles son casa de familia arrendadas, y, por lo mismo estrechas, incómodas e antihigiénicas para el nuevo objeto a que se les destina, que además de los peligros que ofrecen para la salud de los educandos que las habitan, dificultan considerablemente, y aún hacen imposible en gran parte prácticas pedagógicas que deben ser ejecutadas en ella (BOGOTÁ, 1916, p. 68,69).

Aunque en Bolívar, las Escuelas Normales, según el director de la Instrucción Pública del departamento, el doctor Antonio José de Irisarri, contaban para el 1916 con nuevos muebles traídos del extranjero, para 1917 la falta de establecimientos físicos propios para las Escuelas Normales continúa siendo uno de los problemas a resolver por la Nación, el cual se dificulta aún más debido a la crisis fiscal por la que atravesaba el país en la época.

Así lo muestra en 1918 la entonces directora de la Escuela Normal de Institutoras de Bolívar, Ana María Toscano, cuando afirmaba en su informe anual que la institución que aún tenía los muebles suficientes para el cuerpo docente y el área de visitas. De igual forma, manifestaba que a pesar de tener una buena edificación que cumplía con las condiciones higiénicas requeridas y un mobiliario apto para las alumnas-maestras, los dormitorios en inviernos tenían que ser desocupados por causa de inundaciones, lo que, para ella, podría ser la causa de las enfermedades presenten 
en algunas alumnas, puesto que para la fecha se habían retirado tres alumnas-maestras por problemas de salud (BOLÍVAR, 1918).

Un nuevo aspecto, en 1917, es la inclusión de la disciplina de química de los alimentos en los planes de estudio de las Escuelas Normales femeninas. En la ENIB aunque es implementada la asignatura de química, para 1918, la directora manifiesta, de manera imperativa, la necesidad que el Estado suministre los materiales y elementos esenciales para las áreas de física, química e historia de la naturaleza.

De igual forma, se iniciaron cursos de enseñanza de educación infantil y cátedras de pedagogía infantil con la finalidad implementar los kindergardert ${ }^{9}$ en algunas Escuelas Normales del país que se encargarían de la educación para la infancia. En lo que respecta al departamento de Bolívar, en 1916 se crea, anexa a la Escuela Normal de institutoras, una escuela de la infancia, en la que se educan niños de tres a seis años (BOGOTÁ, 1916).

Otra constante para la instrucción pública de la época era el intento por reducir el alto número de maestros y maestras no graduados que laboraban en todo el territorio nacional, puesto que se considerada la formación en el magisterio como el pilar para alcanzar la calidad educativa.

[...] y es el esfuerzo que en todos los Departamentos-en unos más que en otros-viene haciéndose por seleccionar el personal docente de las Escuelas Primarias, sustituyen por Maestros graduados los que no han recibido diploma de idoneidad en alguna de las Escuelas Normales del país. Esta paulatina selección tiene el beneficioso resultado de llevar a las escuelas institutores pedagógicamente para la delicadísima que han de ejercer, a cambios de muchos que por la necesidad o por la mera afición ejercían el magisterio (BOGOTÁ, 1916, p. 5).

Es evidente, la imperiosa labor que el ministro de Instrucción Pública de la época intentaba ejercer para llevar a las escuelas primarias un cuerpo docente preparado y formado que sustituyera al personal no graduado. Sin embargo, esta labor, aunque resaltado en varias Memoria de Instrucción Pública de la época, se contraponía a las constantes reducciones de recursos para la educación y en algunos casos, el cierre de las Escuelas Normales como parte de la solución a la crisis fiscal.

La formación docente se debía ser dada en las Escuelas Normales del país y algunos establecimientos educativos que otorgaban el título de maestros, en trabajo conjunto con la adecuada selección de los profesores para las escuelas primarias. Para 1916 se muestra el cuadro con el total de maestros de cada departamento, destacando graduados y no graduados junto a los sueldos recibidos mensuales. 
Quadro 2 - Numero de maestros y sus sueldos

\begin{tabular}{|c|c|c|c|c|c|}
\hline Departamentos & $\begin{array}{l}\text { Maestros } \\
\text { con grado }\end{array}$ & $\begin{array}{l}\text { Maestros } \\
\text { sin grado }\end{array}$ & $\begin{array}{l}\text { Maestras } \\
\text { con grado }\end{array}$ & $\begin{array}{l}\text { Maestras } \\
\text { sin grado }\end{array}$ & $\begin{array}{c}\text { Sueldo mensual en } \\
\text { pesos oro }\end{array}$ \\
\hline Antioquia & 68 & 304 & 188 & 736 & 10 a 70 \\
\hline Atlántico & 2 & 44 & 12 & 34 & 20 a 70 \\
\hline Bolívar & 20 & 100 & 57 & 95 & 20 a 50 \\
\hline Boyacá & 34 & 136 & 62 & 230 & 3 a 31 \\
\hline Cauca & 26 & 86 & 22 & 130 & 10 a 70 \\
\hline Caldas & 50 & 180 & 43 & 391 & 7 a 65 \\
\hline Cundinamarca & 45 & 37 & 225 & 271 & 10 a 30 \\
\hline Huila & 5 & 35 & 28 & 63 & 20 a 70 \\
\hline Magdalena & 6 & 56 & 5 & 66 & 15 a 50 \\
\hline \begin{tabular}{|l|} 
Nariño \\
\end{tabular} & 25 & 108 & 82 & 100 & 14 a 42 \\
\hline Norte de Santander & 4 & 94 & 24 & 122 & 18 a 25 \\
\hline \begin{tabular}{|l|} 
Santander \\
\end{tabular} & 2 & 55 & 81 & 342 & 12 a 35 \\
\hline \begin{tabular}{|l|} 
Tolima \\
\end{tabular} & 26 & 48 & 28 & 204 & 17 a 70 \\
\hline Valle & 5 & 135 & 28 & 254 & 18 a 60 \\
\hline \begin{tabular}{|l} 
Totales \\
\end{tabular} & 318 & 1.418 & 885 & 3.038 & \\
\hline
\end{tabular}

Fonte: Memoria de instrucción pública, 1916.

En el cuadro anterior es evidente las razones por las cuales el Ministerio de Instrucción Pública mantenía la preocupación por la formación de maestros. En total, el número de profesores y profesoras no graduadas superaba considerablemente el número de los docentes graduados. Para 1916, Colombia tenía en total 5.659 profesores, de los cuales 4.456 eran no graduados y 1.203 poseían título de maestros, lo que indica que solo el 21,25\% de los profesores se habían formado para ejercer la docencia.

Si analizamos el cuadro 2 en cifras de sexo es posible evidenciar que para 1916 el número de maestras tanto graduadas como no graduadas superaba considerablemente el número de maestros. En Colombia, para 1916, el magisterio se consolidaba como una profesión femenina. En total, existían 3.923 profesoras y 1.736 profesores entre graduados y no graduados, lo que indica que las maestras colombianas ocupaban el 69,32\% del magisterio. No obstante, el porcentaje total de maestras graduadas era bajo llegando sólo a ocupar el 22,55\% de la población docente femenina.

Ahora bien, al detenernos en algunas cifras departamentales observamos que el único Departamento en el cual el número de profesores graduados superaba el número de profesores no graduados es el de Cundinamarca, en el que 45 de los docentes hombres eran graduados y 37 de ellos no lo eran. No obstante, esto no se evidenciaba en el número de profesoras, ya que la cifra de las maestras no graduadas era de 271 frente a 225 maestras graduadas en Cundinamarca. Así se identifica que en 1916 en ningún Departamento de Colombia, las maestras graduadas superaban a las maestras nos graduadas.

En lo que respecta al Departamento de Bolívar, el número de profesores graduados llegaba 20 y el de profesores no graduados a 100, lo que indica que sólo el 20\% del cuerpo docente masculino del Departamento de Bolívar había tenido una formación docente completa. En el caso de las maestras podemos observar que las graduadas llegaba a un número de 57 y las no graduadas alcanzaba los 97, demostrando así que las profesoras graduadas superaban el $50 \%$ de aquellas que no poseían formación. Por tanto, es posible afirmar que la formación en la ENIB, a pesar de las falencias expuestas, cumplía positivamente el propósito de educar maestras para ocupar cargos 
en las escuelas primarias y con ello, llegar a "sustituir en la dirección de las escuelas a los inhábiles, a los impreparados y a los desidiosos" (BOGOTÁ, 1916, p. 5).

Otro aspecto a destacar en el cuadro 2 son los salarios de los docentes, los cuales variaban de acuerdo a los Departamentos. Los Departamentos de Antioquia, Atlántico, Cauca, Huila y Tolima presentaban para 1916 los salarios más altos, que oscilaban desde 10 a 70 pesos oros mensuales, siendo Atlántico y Huila los Departamentos con mayores salarios mínimos (20 pesos oros mensuales). No obstante, los Departamentos de Boyacá, Cundinamarca y Norte de Santander poseían los salarios más bajos que iban desde 3 a 35 pesos oro mensuales.

Los maestros del Departamento de Bolívar, según lo indica el cuadro 2, tenían un salario mensual desde 20 a 50 pesos oro. Así mismo, en el informe sobre Tesorería General del Departamento para el mes de diciembre de 1915 se evidencia que los salarios de los maestros, subdirectores y directores de las escuelas bolivarenses variaban desde 15 a 40 pesos oro mensuales. Esto nos parece confirmar que una de las causas para la "feminización del magisterio" estaba intimamente asociada a los bajos salarios pagos a las mujeres.

Si analizamos estos datos con los salarios de 1909, podemos afirmar que 7 años después los maestros de Colombia continuaban obteniendo salarios bajos. El salario mensual desde 1909 a 1916, en el caso específico de Bolívar, había aumentado solo 30 pesos oro, contando que los profesores obtuvieran el monto más alto. Si ocurriera lo contrario, es decir obtuvieran el salario más bajos expuesto en el cuadro 2 , se observa que el salario mensual no había variado en los 7 años.

Por otro lado, para el 1918 se producen dos grandes cambios en las Escuelas Normales. A nivel nacional se suspende el primer año de la formación de profesores y profesoras debido a la crisis fiscal que atravesada el poder ejecutivo. Así, con el Decreto $\mathrm{n}^{\circ} 85$ es suprimido el primer año de formación y con él, se dejan sin labor académica a cinco profesores, por lo que se identifica que las condiciones laborales de los maestros normalistas permanecía en constante cambio, lo que afectaba la estabilidad del cuerpo docente y por ende de la institución.

Según Emilio Ferreiro manifestaba en las Memoria de Instrucción Pública de 1918 era por causa de la crisis fiscal que afrontaba el país en la época que la buena marcha de la Instrucción Pública había sufrido daños (BOGOTÁ, 1918, p. 6). No obstante, Emilio Ferreiro afirmaba que estos daños no habían sido graves.

Que a causa de la crisis fiscal por la que atraviesa el Tesoro Nacional, el Congreso de 1917 autorizó al Poder Ejecutivo, por medio de la ley 51 del mismo año, para suprimir aquellos servicios que a juicio de éste no fueren absolutamente indispensables [...] Artículo $1^{\circ}$ Suspéndese el primer año de estudios en todas las Escuelas Normales de la República, y en consecuencia quedan suprimidos cinco profesores en cada una de dichas escuelas, desde el $1^{\circ}$ de febrero del presente año. Los consejos directivos de las mencionadas escuelas distribuirán las cátedras de los cursos restantes entre los Profesores remunerados y los superiores de estos institutos (BOLÍVAR, 1918, p. 1). 
E1 Decreto n 85 de 16 de enero de 1918 presentaba en dos artículos que en las escuelas normales se debía suspender el primer año de formación. Ello, con el objetivo de prescindir de cinco profesores por escuela normal y ahorrar recursos financieros en el pago de los mismos. Esta medida, en palabras de Emilio Ferreiro, "produjo la eliminación de 123 profesores y 164 becas, con una economía para el Tesoro Público de $\$ 4,305$ cada mes” (BOGOTÁ, 1918, p. 29).

Mas esto no ha sido suficiente: la situación de las Escuelas Normales continuó haciéndose más y más precaria, hasta que llegó el momento en que el Ministerio se vio forzado a disponer que algunas de ellas se convirtieran en externados, porque las contratistas de alimentación, privadas de los recursos que recibían del Gobierno en pago de sus contratos, no pudieron seguir administrándola (BOGOTÁ, 1918, p. 29).

Al ser las escuelas normales administradas por el Tesoro Público Nacional, estas se vieron directamente afectadas con la crisis fiscal del país en el periodo. Las medidas de suprimir el primer año de formación y de convertir algunas de las escuelas normales en externados dificultó el desenvolvimiento que estas instituciones venían realizando desde su creación y dejo sin empleo un gran número de docentes. Ello, nos permite indicar que en situaciones de crisis, desde tiempos anteriores, uno de los primeros recursos suspendidos son los de la educación.

Podemos identificar que las escuelas normales, aún siendo las escuelas formadoras de maestros colombianos, eran las instituciones escolares que más recibían los estragos de las crisis económicas del país. No existían, en la mayoría de las escuelas normales, otros recursos departamentales o municipales que pudieran suplir las faltas del Gobierno.

En el caso particular de la ENIB, según el informe de la Directora de la ENIB en 1918, Ana María Toscano Canal, se administraban aulas en $2^{\circ}, 3^{\circ}$ y $4^{\circ}$ año de formación normalista (BOLIVAR, 1918). Por lo que es posible manifestar que las ordenes expuesta en el Decreto n 85 de 16 de enero de 1918 fueron acatadas por la ENIB. Caso contrario, las escuelas normales del Departamento de Atlántico y la Escuela Normal de la capital del país, Bogotá, no suspendieron el primer año de formación, porque el Gobierno Departamental pudo suplir los gastos escolares (BOGOTÁ, 1918).

Otro de las acciones tomadas fue suprimir los internados. Con relación a ello es posible apreciar que en la ENIB no se aplicó está reglamentación. La Directora Ana María Toscano Canal manifestaba en el mismo informe que existían 32 alumnas internas para el año de 1918 (BOLÍVAR, 1918, p. 48). Sin embargo, se expresaba que el contrato de alimentación no había sido cumplido de manera eficiente debido a la poca pensión alimentaria que la Nación le asignaba a cada alumna.

El señor don Rafael Pereira es el contratista de la alimentación del personal interno de superioras y alumnas. La carestía de los víveres no le permitió cumplir el contrato como él hubiera deseado. Ojalá el Gobierno departamental pudiera conseguir que la Asamblea destinara una partida para aumentar la pensión alimentaria de cada alumno, pues ya es insuficiente la cantidad destinada por la Nación para ese gasto (BOLÍVAR, 1918, p. 50). 
Es notoria la situación de la ENIB con respecto a la alimentación de los empleados y alumnas. Los recursos aportados por la Nación no eran suficientes para suplir la alimentación de la comunidad educativa y por ende, la petición de la directora de la ENIB para aumentar las pensiones alimentarias de los alumnos. Ello, se puede interpretar como una lucha constante de los agentes pertenecientes a la institución por obtener los recursos necesarios para formar profesoras capacitadas.

En el año de 1922, las Escuelas Normales de la República dejan de ser sostenidas por la Nación y pasan a estar a cargo de cada departamento. Para las Escuelas Normales del Departamento del Bolívar se asigna el pago de los alimentos de los catedráticos y los alumnos becados, así como el pago de los sueldos del personal directivo ${ }^{10}$.

Para 1930 se realiza otro cambio importante en las Escuelas Normales. El Decreto $\mathrm{n}^{\circ} 1605$ se considera que los títulos de maestro de escuela elemental y maestro de escuela superior no poseen finalidad práctica y son suprimidos, por lo que sólo se asignan títulos de maestros de escuelas. Para ello, debían cursarse y aprobarse todos los cursos de los cinco años de formación, escribir una tesis sobre un punto pedagógico, preparar tres clases que deberá aplicar en tres aulas distintas.

\section{Consideraciones finales}

Desde su creación hasta la primera mitad del siglo XX, las Escuelas Normales sufrieron cambios significativos, desde el ámbito político, social y económico que orientaron su organización de acuerdo a los cambios de la época. Estos cambios formaron generaciones de profesores que orientaron la educación primaria desde los dogmas del catecismo y el progreso de la Nación y ayudaron a construir las concepciones actuales de lo que es ser maestros, su labor en la escuela y la sociedad.

Sin embargo, las dificultades no fueron ajenas a estas instituciones lo que, de una u otra manera, alteraba su buen funcionamiento. Estas falencias que se hacen presente en la educación colombiana desde hace siglos, es el reflejo de un Gobierno poco interesado por el desarrollo educativo de su país. Así mismo, es evidencia que los recursos educativos han sido constantemente cuestionados, suprimidos o reducidos para suplir otras necesidades, lo que muestra el grado de compromiso e interés por la educación del pueblo.

La Escuela Normal de Institutoras de Bolívar, foco de nuestro análisis, fue una institución que cumplió el propósito de formar maestras para la educación primaria y al igual que la mayoría de las Escuelas Normales del país, padeció los estragos de una Nación dividida políticamente, resistiendo anualmente a los bajos presupuestos, las precarias condiciones higiénicas de las edificaciones y la escasez de recursos escolares. La ENIB llegó a ser una institución no excepta de los cambios político-educativos que los Gobiernos del periodo implementaron en el país al estar manejada por el Tesoro Nacional.

De igual forma, es claro que el compromiso de educar bajo el catecismo se mantiene vigente en la primera mitad del siglo XX. Aunque, también existe un creciente interés por cumplir con las exigencias de la industria y el comercio que en 
la época se incrementan en todo el país. Por ello, los alumnos-maestros de la primera mitad del siglo XX se caracterizan por formarse entre el catecismo, la pedagogía y el conocimiento industrial. Este último, centrado para los hombres.

Estos ideales conservadores marcaron una época en Colombia y con ello contribuyeron a la formación de un ciudadano y una ciudadana guiados por Dios, dedicado al trabajo con miras hacia al progreso. Así mismo, formaron una mujer, hablamos de la clase media, temerosa a Dios, sumida en la obediencia, la caridad y el cuidado al prójimo, capaz de ocupar espacios públicos, siempre y cuando estuviera dentro de los patrones exigidos en la época.

Los países latinos, ejemplo de ellos Colombia y Brasil, presentan similitudes con relación a las Escuelas normales y la educación implementada para formar a las profesoras. Una de ellas, es la imperante presencia de la iglesia católica en la educación y por ende en el magisterio femenino. Así mismo, la concepción de madres y esposas asociadas a la labor de educar se evidencia como uno de los factores que permitieron que las mujeres se ocuparan de labor educativa. Por otro lado, se reafirma que las escuelas normales constituyeron un espacio fundamental para que las mujeres se consolidaran como profesoras y se llevara a cabo la feminización del magisterio.

\section{Referências}

ALMEIDA, J. S. Mulher e educação: A paixão pelo possível. São Paulo: Editora UNESP,1998.

BURKE, P. A Escola dos Annales (1929-1989): a Revolucao Francesa da Historiografia. Traducao Nilo Odalia. 2 Ed. São Paulo: Fundacao Editora da UNESP, 1992, 153 paginas.

BARROS, J. D'A. O campo da história. Santiago: Ediciones UCSH, 2008.

BOGOTÁ. Decreto $\mathbf{n}^{\circ} \mathbf{1 3 1}$ de 1909. Por el cual se organizan las Escuela de Institutores e Institutoras existentes y se crean otras, Revista de Instrucción Pública de Colombia, n 2, 1909.

Cuadro de los maestros y sus sueldos. Memoria del Ministro de Instrucción Pública al Congreso de 1916. Imprenta Nacional, 1916.

Honorables señores y representantes. Memoria del Ministro de Instrucción Pública de 1918. Imprenta Nacional, 1918.

BOLÍVAR. Aviso sobre Instrucción Pública, Diario de Bolívar nº 1725 de 3 de agosto de 1877.

Admisión de las alumnas en la Escuela Normal de Institutoras, Diario de Bolívar $\mathbf{n}^{\circ}$ 1831 del 24 de enero de 1878.

Decreto $\mathbf{n}^{\circ} 194$ de $\mathbf{1}^{\circ}$ de abril de 1905. Organización de los estudios en las Escuelas Normales del departamento. Registro de Bolívar, 1905.

Decreto $\mathbf{n}^{\circ} 38$ de 9 de marzo de 1875. Sobre la Escuela Normal de Institutoras de Bolívar. Diario de Bolívar, n 1086, 1875.

Reformas al reglamento de las Escuelas Normales de la República. Diario de Bolívar $\mathbf{n}^{\circ}$ 554 del 13 de septiembre de 1888.

Visita pasada a la escuela normal de institutoras. Registro de Bolívar, n² 2599, 10 de julio de 1906

Resolución $\mathrm{N}^{\circ} 18$ de 10 de julio de 1918. Expedición de diplomas de maestros en la Escuela Nuestra Señora del Carmen. Gaceta de Bolívar, n² 2444,1918. 
COLOMBIA. Ley Orgánica de Instrucción Pública. Revista de Instrucción Pública, 1927.

FERREIRA, N. V. C. Economia Doméstica: ensino profissionalizantes feminino no triângulo mineiro (Uberaba/MG - 1953-1997). Jundiaí: Paco Editorial, 2014.

FRAGO, A. V. Historia de la Educación e historia cultural. Revista Brasileira de Educação. Brasilia: $N^{\circ}$ 0, págs, 63-82, septiembre de 1995.

HELG, A. La educación en Colombia. 1918-1957. Bogotá: Editorial presencia, 1987.

IBARRA, A. C. Entre la historia y la memoria. Memoria colectiva, identidad y experiencia: discusiones recientes. In: IBARGUEN, M. A.; WALDMAN, G. (Coord.). Memoria (in)cógnitas contenidas en la historia. México, 2007.

LE GOFF, J. Traduccion Bernaldo Leitão. História e memória. Campinas: Editora de la UNICAMP, 1990.

LEVI, G. Sobre a Micro-história. Págs. 133-163. In: BURKE, P. (Org.) Traducción de Magdala Lopes. A escrita da história: Novas perspectivas. $7^{\circ}$ reimpresión. São Paulo: Editora da Universidade Estadual Paulista, 1992.

LOAIZA, G. E1 maestro de escuela o el ideal liberal de ciudadano en la reforma educativa de 1870. Revista Historia Crítica, n. 34. Bogotá, 2007.

LOURO, G. Gênero, sexualidade e educação. Uma perspectiva pósestruturalista. Rio de Janeiro: $6^{\circ}$ ed., Editora vozes Ltda, 2003.

MAGALHÃES, J. P. Tecendo nexos. História das instituições escolares. São Paulo: Editoria Universitária São Francisco, 2004.

MAN, R. La microhistoria como referente teórico-metodológico. Un recorrido por sus vertientes y debates conceptuales. Historia Actual Online. España: n. 30, 2013, p. 167-173.

MÚNEVAR, F. S. Enseñando mutuamente: una aproximación al método lancasteriano y a su apropiación en Colombia. Revista Historia de la Educación Colombia. Pasto: v. 13, n. 13, 2010, p. 47-76, 2010.

NOSELLA, P.; BUFFA, E. Instituições escolares. Por que e como pesquisar. São Paulo: Editora Alinea, 2009.

OBESO, R. Mujer, cultura y sociedad. Barranquilla, 1903-1930. Barranquilla: Fondo de publicaciones de la Universidad del Atlántico, 1999.

PERROT, M. Mi historia de las mujeres. Buenos aires: 1 ed., 1 reimp., Fondo de cultura económica, 2009.

OSPINA, C. El proyecto moderno instruccionista en Antioquia (1903-1930): los modos de Instrucción que funcionaron a instancias de la ley 39 de 1903. Medellín, 2010, 383 p. Tesis (Doctorado en Educación). Universidad de Antioquia, 2010.

OSORIO, M. El surgimiento de las escuelas normales femeninas en Colombia. Revista Historia de la Educación Latinoamericana, Tunja, n. 4, 2002. Recuperado en: <http://revistas. uptc.edu.co/revistas/index.php/historia_educacion_latinamerican/article/view/1471/1466.>. Acceso en: 3 de mayo de 2015.

. Las escuelas normales colombianas y la formación de maestros en el siglo XIX. Revista Eccos, São Paulo, Vol. 7, n. 2, 2005, p. 427-450.

VEIGA, C. G. História da educação. São Paulo: Editora Ática Universitárias, 2007.

VELÉZ, G. Las mujeres en la prensa de Cartagena de Indias 1900-1930. Cartagena: Editorial Universitaria, 2011.

VILLELA, H. de O. S. O mestre-escola e a professora. In: LOPES, E. M. T.; FARIA, F. L.; VEIGA, C. G. (Org.). 500 anos de educação no Brasil. Belo Horizonte: autêntica, 2007, p. 95-135. 


\section{Notas}

${ }^{1}$ El método lancasteriano fue implementado por primera vez en Inglaterra. Según Francisco Sanabria Múnevar (2010) el método lancasteriano o de enseñanza mutua imitaba la organización de las fábricas de textiles; “[...]Se trataba de un gran salón, con un gran número de bancos dispuestos en filas; en el recinto se reunían un maestro, ubicado al frente, y los alumnos, ubicados en las filas. En cada una de las filas, en el extremo, se ubicaba un monitor. El maestro daba la lección únicamente a los monitores, y éstos se la repetían a los demás estudiantes que estaban ubicados en sus respectivas filas [...] (MÚNEVAR, 2010, p. 52). Ver al respecto: MÚNEVAR, F. Enseñando mutuamente: una aproximación al método lancasteriano y a su apropiación en Colombia. Revista Historia de la Educación Colombiana. Pasto: V. 13, N 13, págs. 47-76, 2010

${ }^{2} \mathrm{El}$ término institutora o para el género masculino institutor hace referencia a las maestras y los maestros que se formaban para instituir en las escuelas del país.

${ }^{3}$ A partir de la Escuela de los Annales os estudios sobre la mujer fueron difundidos. Michelle Perrot e George Duby, según Peter Burker (1992), junto a Christiane Klapish, Arlette Farge y Mona Ozouf son algunas de las primeras autoras que se dedican a estudiar la historia de la mujer. Ver al respecto: BURKE, P. A Escola dos Annales (1929-1989): a Revolucao Francesa da Historiografia. Traducao Nilo Odalia. 2 Ed. São Paulo: Fundacao Editora da UNESP, 1992, 153 paginas.

${ }^{4}$ En el año de 1870 con la reforma liberal, Colombia fue constituida como Estados Unidos de Colombia y se dividía geográficamente en 9 Estados Soberanos independientes, entre ellos el Estado Soberano de Bolívar.

${ }^{5}$ En la prensa local de 1878 se encuentran invitaciones realizadas por el secretario general de Estado para construir y donar objetos para Escuela Normal de mujeres de los cuales carece.

${ }^{6} \mathrm{El}$ artículo primero de la Ley Orgánica de Instrucción Pública de 1903 proclama "La Instrucción Pública en Colombia será organizada y dirigida en concordancia con la religión católica”. Ver Revista de Instrucción Pública. Disposiciones vigentes. Disponible en: http://www.banrepcultural.org/sites/default/files/86701/ brblaa499452.pdf. Acceso en: 5 mayo de 2015.

${ }^{7} \mathrm{El}$ partido conservador llega al poder en el año de 1886. A partir de ahí, se empiezan a introducir algunos cambios en las políticas educativas del país. Sin embargo, fue con la Ley Orgánica de Instrucción Pública de 1903 y que estos cambios se organizan.

${ }^{8}$ Con la proclamación de la Constitución Política de 1886, Colombia se organiza como una República y los nueve Estados Soberanos se organizan como departamentos. Así de la fecha, Bolívar es denominado departamento de Bolívar.

${ }^{9}$ Los kindergardert eran establecimientos educativos en los cuales se ofrecía educación a niños entre tres y seis años de edad. Inicialmente se constituyen como escuelas de la infancia anexas a las escuelas normales.

${ }^{10}$ La ordenanza n 46 de 1922 reglamenta que las Escuelas Normales deberán ser sostenidas por el departamento atendiendo a lo estipulado en el decreto $n^{\circ} 319$ que suprime de mantenimiento de estas instituciones por parte del Estado, con excepción de las Escuelas Normales de Bogotá. 
* Mestre em Educação pela Universidade Federal de Mato Grosso, Cuiabá, Mato Grosso, Brasil.

** Professora doutora da Universidade Federal de Mato Grosso, Cuiabá, Mato Grosso, Brasil.

\section{Correspondência}

Nilce Vieira Campos Ferreira - Universidade Federal de Mato Grosso, Instituto de Educação. Avenida Fernando Correa da Costa, 2367, Boa Esperança. CEP: 78060900. Cuiabá, Mato Grosso, Brasil.

E-mail: yesica.montes.g55@gmail.com - nilcevieiraufmt@gmail.com

Recebido em 20 de maio de 2016

Aprovado em 20 de março de 2017 